Published in "Neuropsychological Rehabilitation 26(4): 532-557, 2016"

which should be cited to refer to this work.

\title{
Language specificity of lexical-phonological therapy in bilingual aphasia: A clinical and electrophysiological study
}

\author{
Narges Radman', Lucas Spierer ${ }^{1}$, Marina Laganaro², \\ Jean-Marie Annoni ${ }^{1}$, and Françoise Colombo ${ }^{3}$ \\ ${ }^{1}$ Neurology Unit, Department of Medicine, Faculty of Sciences, University \\ of Fribourg, Fribourg, Switzerland \\ ${ }^{2}$ Faculty of Psychology and Educational Sciences, University of Geneva, \\ Geneva, Switzerland \\ ${ }^{3}$ Neuropsychology Unit, Hôpital fribourgeois, Fribourg, Switzerland
}

Based on findings for overlapping representations of bilingual people's first (L1) and second (L2) languages, unilingual therapies of bilingual aphasia have been proposed to benefit the untrained language. However, the generalisation patterns of intra- and cross-language and phonological therapy and their neural bases remain unclear. We tested whether the effects of an intensive lexical-phonological training (LPT) in L2 transferred to L1 word production in a Persian-French bilingual stroke patient with Broca's aphasia. Language performance was assessed using the Bilingual Aphasia Test, a 144-item picture naming (PN) task and a word-picture verification (WPV) task. Electroencephalography (EEG) was recorded during PN and WPV in both languages before and after an LPT in French on a wordlist from the PN task. After the therapy, naming improved only for the treated L2 items. The naming performance improved neither in the untrained L2 items nor in the corresponding items in L1. EEG analyses revealed a Language $\mathrm{x}$ Session topographic interaction at $540 \mathrm{~ms}$ post-stimulus, driven by a modification of the electrophysiological response to the treated L2 but not L1 items. These results indicate that LPT modified the brain networks engaged in the phonological-phonetic processing during naming only in the trained language for the trained items.

Correspondence should be addressed to Narges Radman, Neurology, Department of Medicine, University of Fribourg, Chemin du Musée 5, CH-1700 Fribourg, Switzerland. E-mail: narges.radman@unifr.ch 
Keywords: Bilingual aphasia; Lexical-phonological therapy; Cross language generalisation; Brain lesion.

\section{INTRODUCTION}

The term "bilingual" refers to speakers being proficient in at least two languages. Clinical and neuroimaging data indicate that in bilinguals, partly shared brain structures support lexical and morphosyntactic representations of the first (L1) and the second language (L2) (Goral, Levy, \& Kastl, 2007; Kroll \& Stewart, 1994), with the degree of overlap mostly depending on proficiency in the second language.

Because of the partial overlap between L1 and L2 representations, brain lesions in bilingual speakers may alter the two languages in parallel or differently (Lucas, McKhann, \& Ojemann, 2004). Importantly, post-lesional patterns of language recovery may also vary (Paradis, 1998), with (1) both impaired languages improving to a similar extent and concurrently (parallel recovery); (2) one language recovering better (differential recovery, usually the first language); (3) one language remaining impaired while the other recovers (selective recovery); and (4) the complete recovery of one language preceding the recovery of the other language (successive recovery). Other clinical reports coming from bilingual aphasics using languages with substantial structural differences suggest that type and degree of language mastery as well as many cultural and language-specific factors influence the recovery of one or the other language (Aglioti, Beltramello, Girardi, \& Fabbro, 1996; Gil \& Goral, 2004; Nilipour, 1988).

Since the majority of the world's population is becoming bilingual, the number of bilingual aphasic patients is rapidly growing (Faroqi-Shah, Frymark, Mullen, \& Wang, 2010). However, the optimal language rehabilitation strategy for bilingual aphasic patients remains unclear. Empirical data enabling the establishment of principled bases upon which to decide whether therapies of bilingual aphasic patients should focus on the rehabilitation of one or both languages, and whether the first or the second language should be rehabilitated, are lacking (Ansaldo \& Saidi, 2014).

Despite the fact that conducting therapy in both languages has been advanced to facilitate language recovery (Ansaldo, Marcotte, Scherer, \& Raboyeau, 2008; Kohnert, 2004), the general trend in aphasia rehabilitation still favours "monolingual" therapies (i.e., a therapy in one language) for the following reasons: (1) bilingual therapy has been argued to confuse the patient and lead to an increase in code mixing or code switching or that improvement occurs in only one of the treated languages (Edmonds \& Kiran, 2006; Kiran, Sandberg, Gray, Ascenso, \& Kester, 2013); (2) bilingual 
therapy can often not be provided due to practical limitations, and (3) based on evidence that in bilinguals the two languages usually share the same lexical and morphosyntactic representations (Gollan, Montoya, FennemaNotestine, \& Morris, 2005), unilingual therapy may be the optimal approach to improve both languages in bilingual aphasic patients because crosslanguage treatment generalisation effects (CLG) should occur (Faroqi-Shah et al., 2010; Kohnert, 2009).

CLG effects are reported to depend on several variables: similarities between languages and words, premorbid language proficiencies, treated language, cultural aspects, intensity of therapy, etc. Intra-language generalisation has already been observed in studies on naming therapy for withinlanguage anomia. In these studies, semantic therapies (a word retrieval treatment relying on the semantic attributes of objects) have been shown to generalise to untreated items, whereas the effects of phonological therapy (i.e., a word-retrieval treatment strategy based on phonological cueing) have generally been reported to be item-specific (Howard, Patterson, Franklin, Orchard-Lisle, \& Morton, 1985).

Concerning cross-language generalisation patterns induced by semantic or phonological therapies, semantic therapy has been reported to induce CLG in studies with bilingual aphasic patients (Croft, Marshall, Pring, \& Hardwick, 2011; Edmonds \& Kiran, 2006; Kiran et al., 2013; Kohnert, 2004; Miertsch, Meisela, \& Isel, 2009). In contrast, although Hinckley (2003) and Marangolo et al. (Marangolo, Rizzi, Peran, Piras, \& Sabatini, 2009) showed some level of CLG with mixed semantic-phonological therapy or using phonological therapy in L2, CLG was not found after phonological therapy in Meinzer et al. (Meinzer, Obleser, Flaisch, Eulitz, \& Rockstroh, 2007), or transfer was limited to phonologically similar words (cognates) (Kohnert, 2004; Pillon \& de Partz, 2005). CLG is also influenced by the lexico-semantic organisation in bilingual speakers, which depends on the level of convergence between language representations (Abutalebi \& Green, 2007) and lexical processing (Parker Jones et al., 2012).

Although specific CLG patterns have been reported in the literature reviewed above, whether and how lexical-phonological therapy in L2 would induce intra- and cross-language generalisation and the effects of such therapy on the brain mechanisms involved in naming in L1 and L2, remain largely unresolved. Specific predictions on CLG after lexical-phonological therapy in L2 could however be made based on current models of the bilingual lexical organisation and access.

Regarding the organisation of the bilingual lexicon, we will first summarise word production models (mainly based on picture naming studies). Such models agree that the following processing stages are involved in word production: semantic system activation from a concept, activation of the corresponding lexical nodes (the lemma in some models; Levelt, Roelofs, \& 
Meyer, 1999), retrieval/activation of the phonological representations, and finally motor planning for articulation.

According to a majority of models of bilingual lexical organisation, the two languages share the same semantic system (Costa, Colomé, \& Caramazza, 2000). Differences appear between models mostly at the lexical/phonological level. Some models propose that the activation of this shared semantic system spreads only to the lexical representations of the languages in use (language-specific lexical selection; Costa et al., 2000; Roelofs, 1998), while other theories suggest that the activation of this shared semantic system spreads to the two lexicons, i.e., parallel activation of the two languages (Language non-specific lexical selection; De Bot, 1992; Hermans, Bongaerts, de Bot, \& Schreuder, 1998). In the phonological retrieval stage, two different views have been discussed (see details in Costa et al., 2000): According to the discrete view, only the phonological segments of the selected word are activated and the activation of non-selected lexical representations does not spread to their phonological segments. In contrast, the cascaded view states that activation spreads from all lexical nodes (selected and non-selected) to their phonological representations. In this regard, some authors (Costa et al., 2000; Peterson \& Savoy, 1998) suggest that the activation of lexical representations of the non-target language spreads to their phonological representations. Accordingly, language-specific lexical selection predicts no transfer of the effect of therapy to the untreated language, while language non-specific lexical selection stands for a transfer of the effect of therapy to the untreated language (because of the flow of activation of the semantic system to the lexical representations in both languages). More specifically, if the "discrete view" of phonological retrieval is correct, we should not expect the transfer of the effect of therapy to untrained items. In contrast, the "cascade view" predicts the transfer of the effect of therapy to untrained items.

Among the cascade view models, two make specific predictions on CLG. The Bilingual Interactive Activation + model $(\mathrm{BIA}+)$ predicts important CLG effects with phonological therapy (Dijkstra \& van Heuven, 2002). This model indeed suggests that an integrated lexicon stores all the words across languages and is accessed in a language non-selective way (parallel activation of both languages) and, thus, modifying the lexicon by training phonology in one language should impact on the untrained language, especially when the two languages are phonologically similar. The Revised Hierarchical model enables even more specific predictions on the CLG, induced by unilingual phonological therapy: since the different languages have different although interconnected lexicons with stronger associations from L2 to L1, a phonological therapy in L2 is more likely to generalise to L1 compared to therapy in L1. In addition, since the lexicon interacts with semantic representations, lexico-semantic processing should also be 
improved with phonological therapy and in turn improve the global score of clinical aphasia assessment (Faroqi-Shah et al., 2010; Kroll \& Stewart, 1994).

To test these hypotheses, the present study investigates the behavioural and electrophysiological cross-language generalisation of the effects induced by an intensive lexical-phonological therapy in L2 in a bilingual patient (L1: Persian, L2: French), who suffered from initial global aphasia with evolution to Broca's aphasia following left fronto-temporo-parietal ischaemic stroke. We tested the effect of therapy specifically on naming, but also on the word-picture matching as a semantic control task. The rationale for choosing the type and language of therapy were: (1) the patient had a Broca's aphasia with important apraxia of speech and anomia but only mild comprehension deficits, and therefore her word-finding difficulty was considered to be related to post-semantic levels; (2) the patient was professionally active in an L2 environment before the stroke and the main language in her everyday life was French (L2). Based on the language-specific lexical selection model, the effect of therapy should not transfer to the untreated language, while the language non-specific lexical selection model predicts generalisation to the untreated language. However, given the limited lexical similarity between Persian and French, two languages with few cognates, we predict that lexical-phonological therapy in L2 should not transfer to the untreated language. We used eventrelated potential (ERP) analyses during the word-picture matching and the picture naming tasks to identify whether and how the therapy impacted the temporal dynamic of lexical-phonological processing of our patient. ERP analyses can indeed provide detailed temporal information on the modifications of brain processing induced by the therapy. Based on current models of the dynamics of picture naming (e.g., Indefrey, 2011), the latency of the ERP modifications following therapy can help determine whether visuo-perceptual, lexical-semantic and/or phonological-phonetic stages were modified.

\section{METHODS}

\section{Case report}

\section{Patient's history of bilingualism}

The patient KJ was born in Iran and had Iranian parents. Her maternal language (L1) was Persian (Farsi), and she had started architectural studies in Iran, although she had to quit her university studies after 1.5 years. She started to learn French at the age of 26 when she moved to a French-speaking part of Switzerland. She had worked in a French-speaking environment for about 20 years before the stroke. Her language use was mainly in French; she spoke in French at work (100\%), 50\% French, 50\% Persian with her 
children and $100 \%$ in Persian with her siblings and parents. She followed TV and radio programmes in French. However, her reading was mainly in Persian; she read newspapers in French (30\%), and books and journals in Persian (70\%).

The patient had also learnt English at school at the age of 12 and she had used English at University (for some courses). According to the patient, her English performance was poor and she used English very rarely. For this reason, although not congruent with the temporal order of learning French and English, we considered French (the main language of daily use) as her second language (L2) and English as her third language (L3). The performance in L3 was not evaluated (Grosjean, 2004).

\section{Clinical history}

Patient $\mathrm{KJ}$ is a 52-year-old right-handed bilingual well-educated woman working as a healthcare aid. She had no significant past medical history and was admitted at Lausanne University Hospital (CHUV) mute and with right sensorimotor hemi-syndrome. The initial cerebral CT scan suggested a left sylvian ischaemic stroke due to the occlusion of the first segment of the left middle cerebral artery (M1). A rapid clinical language examination in the emergency room revealed a global aphasia without any oral expression, reduced written abilities, and comprehension impairment. Two hours after the acute event, she received intravenous thrombolysis and a mechanical thrombectomy. However, the placement of a stent failed because of an important vasospasm. The patient was then transferred to Fribourg Cantonal Hospital.

She spent the first week in the stroke unit where she benefited from standard medical procedures for cerebrovascular accident management, as well as speech and language therapy, physiotherapy and occupational therapy. She was then transferred to the neurorehabilitation unit where the initial language evaluation showed a persistent severe Broca's aphasia; she could only produce some isolated speech sounds, presented a complete failure in picture naming, and there was no automatic-voluntary dissociation. Auditory and written comprehension was only slightly disrupted. She also presented agraphia and oro-facial and speech apraxia. Brain MRI performed three months after the stroke confirmed the sequel of the left sylvian ischaemic stroke without any sign of bleeding in the stroke site nor new ischaemic stroke (Figure 1). Two weeks after the stroke, an evaluation was performed in French. A general rehabilitation programme was started; the patient underwent 12 weeks of rehabilitation for a total of $43 \times 45$-minute sessions with global multimodal therapy in French (L2), including semantic classification, naming, cueing, reading and writing tasks. During this period, comprehension and pragmatic communication improved, and she started to express short words but was still impaired by her speech apraxia and word finding difficulties. Table 1 shows language performances over three evaluations: at the 


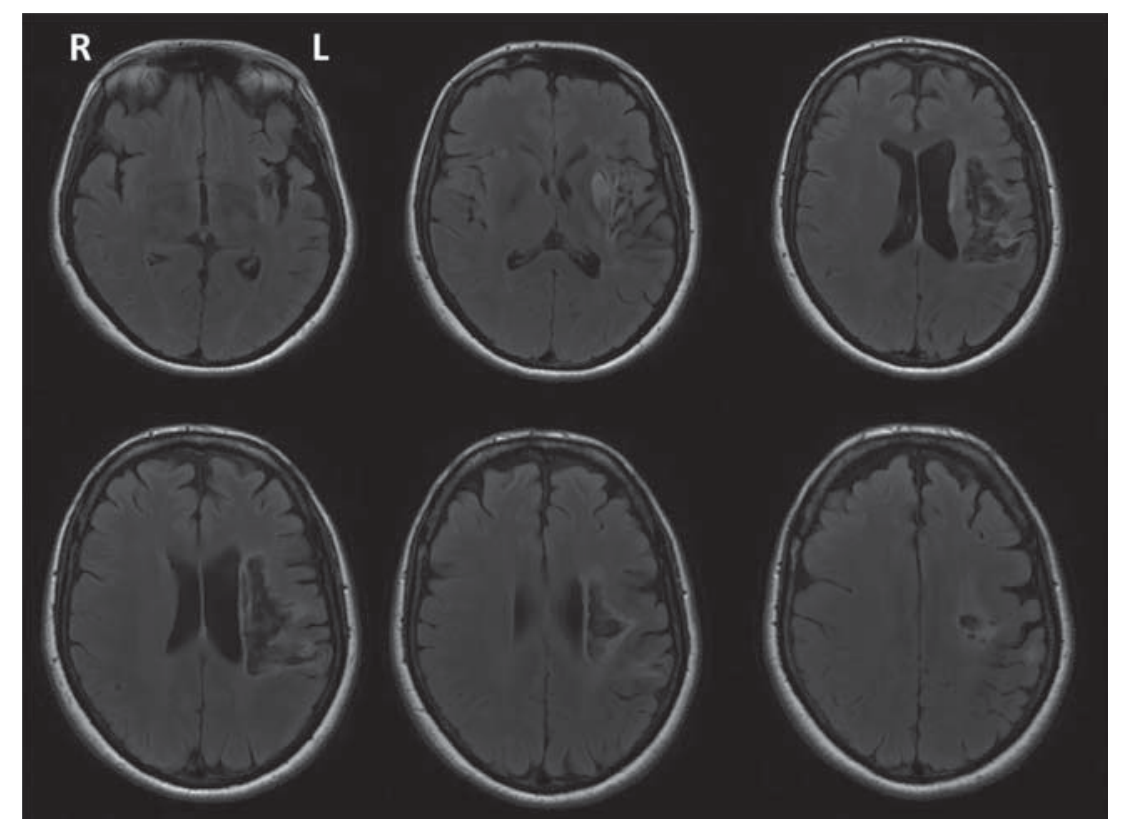

Figure 1. T2-FLAIR MRI sequences at the chronic stage: Brain MRI performed 3 months after the stroke confirmed the sequelae of the left sylvian ischaemic stroke (with the involvement of left frontotemporo-parietal regions) without any sign of bleeding in the stroke site nor new ischaemic stroke.

acute phase (week 2, French), T1 (week 16, before the start of therapy, both languages) and T2 (after the therapy, both languages). The acute phase evaluation was performed only in French.

\section{Experimental session}

We investigated the behavioural and electrophysiological effects of a lexicalphonological intensive computer-assisted therapy conducted in L2 on L1 and L2. The first session of this study consists of the evaluation of "picture naming" performance 15 weeks after the stroke (T0). The complete behavioural assessments and the EEG recording were performed before (T1: 16 weeks after the stroke) and after a four-week intensive phonological speech therapy programme (T2) (Figure 2).

\section{Evaluation of languages pre- and post-phonological therapy sessions}

Behavioural language assessment at TO. Picture naming was assessed using a 144-item task composed of matched sets of items for French 
TABLE 1

Language performance at week 2, T1 and T2, Bilingual Aphasia Test (BAT)

\begin{tabular}{|c|c|c|c|c|c|c|c|}
\hline & \multirow{2}{*}{$\begin{array}{c}\begin{array}{c}\text { French } \\
\text { only }\end{array} \\
\text { Week } 2\end{array}$} & \multicolumn{3}{|c|}{ Persian (L1) } & \multicolumn{3}{|c|}{ French (L2) } \\
\hline & & $T 1$ & $T 2$ & $\begin{array}{c}\mathrm{p}- \\
\text { value }\end{array}$ & $T 1$ & $T 2$ & $\begin{array}{c}\mathrm{p}- \\
\text { value }\end{array}$ \\
\hline Verbal comprehension $^{\mathrm{a}}$ & $6 / 40$ & $32 / 38$ & $40 / 41$ & $0.031^{*}$ & $37 / 40$ & $38 / 43$ & 1 \\
\hline $\begin{array}{l}\text { Syntactic } \\
\text { comprehension }\end{array}$ & $6 / 10$ & $27 / 47$ & $44 / 47$ & $.000^{*}$ & $29 / 43$ & $35 / 47$ & $.031^{*}$ \\
\hline Reading comprehension & $2 / 5$ & $12 / 20$ & $16 / 20$ & .125 & $12 / 20$ & $12 / 20$ & 1 \\
\hline Semantic category & - & $5 / 5$ & $5 / 5$ & 1 & $5 / 5$ & $5 / 5$ & 1 \\
\hline Grammatical judgement & - & $10 / 10$ & $10 / 10$ & 1 & $7 / 10$ & $5 / 10$ & .5 \\
\hline Semantic acceptability & - & $10 / 10$ & $10 / 10$ & 1 & $10 / 10$ & $10 / 10$ & 1 \\
\hline $\begin{array}{l}\text { Word non-word } \\
\text { repetition }\end{array}$ & $0 / 4$ & $17 / 30$ & $29 / 30$ & $.000^{*}$ & $19 / 30$ & $23 / 30$ & .125 \\
\hline $\begin{array}{l}\text { Word non-word } \\
\text { judgement }\end{array}$ & - & $20 / 30$ & $30 / 30$ & $.002^{*}$ & $26 / 30$ & $27 / 30$ & 1 \\
\hline Series & Unable & $0 / 2$ & $1 / 2$ & .24 & $0 / 2$ & $1 / 2$ & .24 \\
\hline Mental arithmetic & $\begin{array}{l}\text { No } \\
\text { response }\end{array}$ & $4 / 6$ & $6 / 7$ & .5 & $4 / 6$ & $2 / 7$ & .5 \\
\hline Reading & r- & $7 / 10$ & $9 / 10$ & .5 & $0 / 10$ & $5 / 10$ & .063 \\
\hline Copying & Her name & $5 / 5$ & $5 / 5$ & 1 & $5 / 5$ & $5 / 5$ & 1 \\
\hline Dictation & $0 / 2$ & $1 / 5$ & $1 / 5$ & 1 & $0 / 3$ & $0 / 5$ & 1 \\
\hline Global score & & $159 / 218$ & $206 / 222$ & $.000^{*}$ & $154 / 214$ & $168 / 224$ & .250 \\
\hline
\end{tabular}

${ }^{\mathrm{a}}$ Verbal comprehension tests consisted of: Pointing, simple and semicomplex orders, complex orders and verbal auditory discrimination. Evaluation of week 2 was performed only in French and according to the patient's performances.

${ }^{*} p<.05$ considered significant.

(D144) (Laganaro, Di Pietro, \& Schnider, 2003, 2006) (see section on Experimental stimuli and tasks, below). As the main focus of the present study is to evaluate the possible cross-language generalisation of a lexical-phonological therapy, assessments for the target picture-naming task were done twice at baseline before therapy (T0 and T1) and once after therapy (T2), in L1 and L2 in different sessions two days apart. EEG acquisition was performed during D144 picture-naming and word-picture verification tasks at T1 and T2 (see below).

Behavioural language assessment at T1 and T2. The patient's language functions were assessed using some subtests of the Bilingual Aphasia Test (BAT, part B) in Persian (L1) and French (L2) on separate non-consecutive days, i.e., with one day in between, for different language modalities. These evaluations were performed by native Persian and French speakers. 


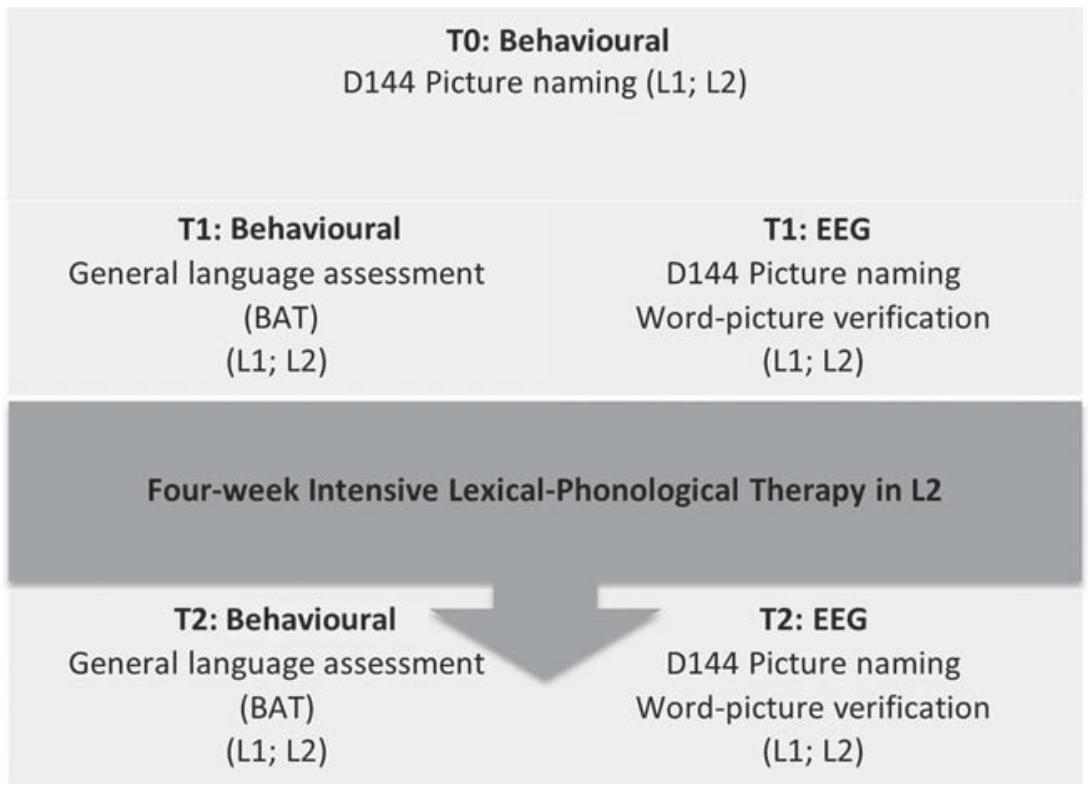

Figure 2. Study design: Both languages (L1; L2) were evaluated behaviourally and with EEG recording before (T1) and after (T2) an intensive lexical-phonological therapy in L2.

Scoring was performed based on the BAT scoring guidelines (Paradis \& Libben, 1987).

The BAT is a comprehensive language test designed to assess the main language functions and to compare patients' performance in their spoken languages (e.g., spontaneous speech, oral production, comprehension). The BAT is a "criterion-referenced" test: based on the test design, the success criterion score should be as close as possible to $100 \%$ correct for each subtest (Paradis \& Libben, 1987).

A picture-naming task (D144) (Laganaro et al., 2003, 2006) and wordpicture verification task were performed before (T1) and after (T2) an intensive phonological language therapy in L2 (French). EEG acquisition was performed during D144 picture-naming and word-picture verification tasks at two phases.

\section{EEG study}

Experimental stimuli and tasks.

- Picture naming: The 144 naming task consisted of two parallel lists of 72 items selected from previous studies (Laganaro et al., 2003, 2006); one 
list was selected to be treated and the other as a control list. The items in the two lists were matched for name agreement, lexical frequency, word length and syllabic structure in French. In the absence of lexical values for Persian words, a Persian version was created by translation of the French stimuli by a native Persian speaker. The translation was controlled by five native Persian speakers and further adapted based on the results of testing of five age- and education-matched Persian speakers. The D144 list consisted of 20 cognate words. The number of phonemes was significantly higher in Persian $(5.75 \pm 2.3)$ than in French $(4.1 \pm 1.5), p<.001$. However, the words were longer in French (word length: $6.02 \pm 1.8$ ) than in Persian (word length: $4.61 \pm 1.6$ ), $p<.001)$.

- Word-picture verification task: The stimuli consisted of 96 image/word pairs. The images were selected from the same database as for the picture-naming task. Half of the image/word pairs were matched, the other half were unmatched. Half of the total word/image pairs were selected from the treated list and the other pairs were selected from the untreated list.

\section{Procedure and task.}

- Picture-naming task: The patient was instructed to name the pictures which were presented for 2 seconds at the centre of a 15" LCD screen with a refresh rate of $60 \mathrm{~Hz}$. Each picture was preceded by a fixation cross for $500 \mathrm{~ms}$ and a grey colour screen for $200 \mathrm{~ms}$ and followed by a white colour (response) screen of a variable length (defined by the experimenter between 3000 and $8000 \mathrm{~ms}$ ) and a grey screen for $200 \mathrm{~ms}$. (Figure 3). Each trial was started manually by the experimenter. The same 144 images were presented for both the French and Persian sessions in different pseudo-random orders. Each language was tested on a separate day so that there was a total of four days with an EEG recording (L1 and L2 at T1 and T2). Each experimental recording consisted of two blocks. The experiment lasted about 40 minutes with a 5minute pause between blocks. To score the patient's naming performance, only first-attempt correct responses within 5 seconds of the presentation of the image was scored as correct. Phonetic approximations (phoneme distortions leading to intelligible words) were considered as correct responses. No-responses, phonological and semantic transformations and a response in an undesired language (switch) were considered as errors.

- Word-picture verification task: Each trial started with a written word presented on the screen for $3500 \mathrm{~ms}$, followed by a fixation cross of 


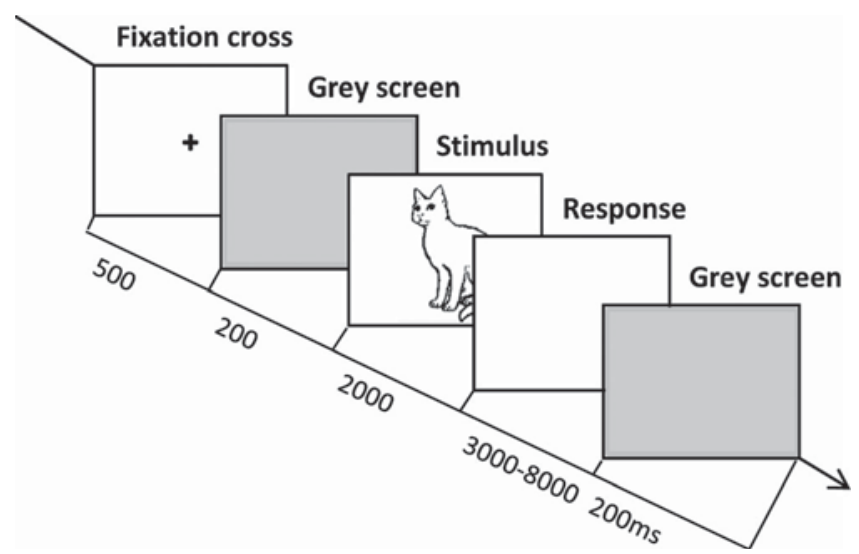

Figure 3. Picture-naming paradigm: Picture-naming task. Each picture was preceded by a fixation cross and a grey colour screen and followed by a white colour (response) screen of a variable length, defined by the experimenter, and finally a grey screen. The timing is indicated in the figure. Each trial was started manually by the experimenter.

$500 \mathrm{~ms}$, a grey screen for $200 \mathrm{~ms}$, a target picture for $2000 \mathrm{~ms}$, and a blank page for $200 \mathrm{~ms}$. The patient had to press a button to respond as soon as the picture appeared on the screen. The responses were recorded if they were performed up to $2200 \mathrm{~ms}$ after the onset of the stimulus presentation. In 48 trials, picture and image were matched and in the other 48 trials they were not matched. The patient was asked to press the button "Y" when the picture and word matched and to press "N" where they did not match. The same paradigm was performed in French and Persian in the corresponding picture-naming session.

During the EEG recording in each language session, the two experimenters talked only the target language to prevent the participant from being in "bilingual" mode (speaking only in French on the French testing days and in Persian on the Persian testing days; Grosjean, 2004).

The entire sessions were audio-recorded using a digital voice recorder. Picture-naming scoring was done online and subsequently double-checked based on the audio-recording. The word-picture verification task was scored offline using E-prime outputs. The E-Prime 2.0 software (Psychology Software Tools, Sharpsburg, PA) was used to deliver stimulations and collect manual responses.

\section{Lexical-phonological computer-assisted therapy}

Sixteen weeks after the stroke, the day after our second pretest baseline, the patient received an intensive lexical-phonological computer-assisted therapy 
in L2 (French). A period of intensive (4 weeks, 5 days per week) phonological therapy of naming in L2 was conducted using a computerised aphasia therapy (CAT) for anomia. Of note, this protocol has already been used with monolingual French-speaking patients (Laganaro et al., 2006).

The lexical-phonological therapy consisted of $5 \times 1$-hour sessions in the first week, and $15 \times 45$-minute sessions afterwards (based on the patient's request to decrease the duration of each session). The patient received a total of 16 hours of therapy.

During this therapy, only one list of 72 French words was treated. The treated words were selected among the items of the D144 picture-naming task (see section on stimuli above). Two tasks were used, involving limited computer skills (the patient had to use the keyboard for writing or copying and the mouse for selecting a button on the screen). In the first programme, a spoken-written picture-naming task, the patient had to write the word corresponding to a picture which appeared on the screen. Two help buttons were available: one provided the pronounced word (phonological cue), the second provided help on each letter of the word (orthographic cue). The second task consisted of a spoken naming with first grapheme help as well as the pronounced word.

\section{Behavioural data analyses}

In order to test the interaction between the factors Language and Session and the main effect of these factors on picture-naming and word-picture verification for the treated and untreated items, the scores were subjected to separate generalised linear mixed effect regression (GLMER) analyses for binomial data using R (Team, 2008), lme4 (Bates, Maechler, Bolker, \& Walker, 2014) and contrast (Kuhn, Weston, Wing, Forester, \& Thaler, 2013) packages. For the fixed effects, we included Language and Session as categorical variables, an interaction term, and we included the items as random effect.

\section{EEG analyses}

\section{EEG acquisition and pre-processing}

The EEG was recorded with a sampling rate of $512 \mathrm{~Hz}$ from 64 electrodes (64-channel ActiveTwo system from Biosemi, Inc., Amsterdam, The Netherlands). The ground and reference electrodes were placed at the inion and vertex ("Cz"), respectively. The data were analysed off-line using the Cartool software (http://brainmapping.unige.ch/Cartool.php) (Brunet, Murray, \& Michel, 2011) as well as the Ragu software (Koenig, Kottlow, Stein, \& Melie-Garcia, 2011). Epochs from $-100 \mathrm{~ms}$ before the presentation of the stimuli to $600 \mathrm{~ms}$ post-stimulus were extracted from the raw EEG data 
and filtered between 0.1 and $30 \mathrm{~Hz}$ with the addition of a $50 \mathrm{~Hz}$ Notch filter to remove $\mathrm{AC}$ noise. Epochs with eye-blinks or other artefacts (as determined by amplitude changes exceeding $80 \mu \mathrm{V}$ on at least one electrode during the epoch) were rejected before epoch averaging. For both paradigms, only epochs corresponding to the treated items (independently of the patient's response), accepted in all four conditions were considered, leading to the inclusion of 36 out of 144 epochs for picture naming and 33 out of 96 epochs for word-picture verification. The epochs were then averaged separately for each of the experimental conditions and recomputed against the average reference.

\section{Event-related potentials}

A first level of analysis was conducted by comparing the ERPs in the L1/ $\mathrm{T} 1 ; \mathrm{L} 1 / \mathrm{T} 2, \mathrm{~L} 2 / \mathrm{T} 1$ and L2/T2 conditions using a time-frame wise $2 \times 2$ within-subject ANOVA with factors Language (L2; L1) and Session (T1; T2) at each scalp electrode as a function of peri-stimulus time. The results of this ERP waveform analysis are presented as a plot (Figure $5 \mathrm{~b}$ ) depicting the time frames showing a significant $(p<.01)$ Language $\times$ Session interaction as a function of peri-stimulus time and electrodes. While highly sensitive, the statistical results of the ERP analyses are dependent on the choice of the reference electrode. We therefore base our interpretations on referenceindependent global analyses of the topography of the electric field at the scalp.

\section{Global dissimilarity analysis}

Topographic modulations were analysed using randomisation statistics applied to Global Map Dissimilarity measures (GMD; Lehmann \& Skrandies, 1980) using a $2 \times 2$ within-subject ANOVA with factors Language (L1; L2) and Session (T1; T2). GMD is calculated as the root mean square of the difference between the strength-normalised voltage potentials across the electrode montage. We analysed GMD values as a function of peri-stimulus time as for the ERP analyses (Koenig et al., 2011; Koenig \& Melie-Garcia, 2010; Murray, Brunet, \& Michel, 2008). Correction was made for temporal autocorrelation through the application of a $>11$ contiguous data points temporal criterion for the persistence of significant effects (Guthrie \& Buchwald, 1991). In addition to the independence on the choice of the reference electrode, the GMD global analyses of the shape of the electric field over local electrode analyses allows for neurophysiological interpretation of the observed effects. Because topographic modulations necessarily follow from modifications in the configuration of the underlying neural generators, GMD modulations indicate that qualitative changes in the brain networks engaged across conditions (Lehmann, Ozaki, \& Pal, 1987). As 
strength-normalised maps are used in the calculation on the GMD, explanation of our results in terms of pure amplitude modulation can be ruled out.

\section{RESULTS}

\section{Behavioural results}

\section{General language assessment}

The patient was evaluated using the L2 (French) and L1 (Persian) versions of BAT, before and after the language phonological therapy (T1; T2). Table 1 shows the detailed result of the patient's performance.

At T1, the overall pattern based on the BAT results showed no difference between L1 and L2 (McNemar's chi-squared $p=1.0$ ). Before the therapy at $\mathrm{T} 1$, the major impairment for L1 was found in the following subtests: semicomplex orders, verbal-auditory discrimination, syntactic comprehension, repetition, series, mental calculation, word reading, dictation and words and phrases comprehension.

At the same phase (T1), the patient was below the limits of the norm in L2 for complex orders, syntactic comprehension, acceptability judgement, word and nonword repetition and judgement, series, naming, mental calculation, word reading, dictation and word and phrase comprehension.

After the therapy (at T2), in the BAT, her global score of L1 improved. Specifically, the scores in verbal comprehension and word and nonword repetition and judgement in L1 significantly improved at T2. She also performed better in syntactic comprehension in both languages at T2. However, her global score in L2 function did not improve after the therapy (Table 1).

\section{D144 picture naming}

Pre-treatment picture-naming scores (at T0 and T1, i.e., one week apart) were analysed to check for the amount of variability of language performance immediately before starting the therapy. A GLMER model was applied to analyse the interaction between the factors Session (T0; T1), Language (L1 untreated; L2 treated) and List (treated items; untreated items). The results showed a main effect of factor Language $(z=-2.70, p=.006)$, a main effect of Session $(z=-2.5, p=.012)$ (T0 $>\mathrm{T} 1)$, but there was no main effect of List $(z=0.32, p=.74)$. No interaction was found between the factors Session and List $(z=-1.03, p=.30)$, nor between Language and List $(z=0.31, p=.75)$. However, an interaction was found between Language and Session $(z=2.74, p=.006)$. This interaction was driven by a "trend" to decrease in L1 naming score from T0 to T1, $z=-2.01$, 
$p=.08$ (Bonferroni corrected) but no change in $\mathrm{L} 2$ naming from $\mathrm{T} 0$ to $\mathrm{T} 1, z=$ $-0.611, p=1.0$ (Bonferroni corrected). See Figure 4(a).

A GLMER model was applied to analyse the interaction between the factors Session (T0; T1) and Language (L1 untreated; L2 treated) for the treated and untreated items separately.

Regarding the treated items, there was no main effect of Session $(z=0.20$, $p=.84)$, but there was a main effect of Language $(z=4.50, p<.001)$, and an interaction between the factors Language and Session $(z=4.79, p=.03)$. The interaction was driven by an improvement in the treated $\mathrm{L} 2, z=-2.77$, $p=.01$ (Bonferroni corrected), Cohen's $d=4.71$, but not in the untreated L1 language, $z=0.19, p=1.0$ (Bonferroni corrected), Cohen's $d=-0.79$. The effect size (Cohen's $d$ ) of the therapy on all items was $d=5.42$ for the treated language (L2) and $d=-0.87$ for the untreated language (L1).

Testing the untreated items, there were no main effects of factors Session $(z=0.37, p=.71)$ and Language $(z=-0.19, p=.85)$. Moreover, no interaction between the factors Language and Session was found $(z=-0.39, p=$ .69). See Figure 4(a).

The number of semantic errors (7\% of errors in L1 and 2\% in L2), phonological errors ( $24 \%$ of errors in L1 and $25 \%$ in L2), and switches (4\% of errors in $\mathrm{L} 1$ and $1 \%$ in L2) as well as the other types (including no responses and late responses) in picture naming was similar at T1 in both languages. Similarly, the number of semantic errors ( $6 \%$ of errors in $\mathrm{L} 1$ and $3 \%$ in $\mathrm{L} 2$ ), and switches ( $4 \%$ of errors in $\mathrm{L} 1$ and $1 \%$ in L2) were similar at $\mathrm{T} 2$ in both languages, and there was a larger number of phonological errors (although not statistically significant) in L2 (35\% of errors in L2) than and in L1 (25\% of errors in L1) $p=.37$.

\section{Word-picture verification task}

The same GLMER model as for the picture-naming task was applied to analyse the interaction between the factors Session (T1; T2) and Language (L1 untreated; L2 treated) for the treated and untreated items separately.

Regarding the treated items, there was no main effect of Session $(z=$ $-0.37, p=.70)$ or Language $(z=0.70, p=.48)$. There was also no interaction between the factors Language and Session $(z=-0.43, p=.66)$.

For the untreated items, there was no main effect of Session $(z=-0.85$, $p=.39)$ or Language $(z=1.14, p=.25)$. There was also no interaction between the factors Language and Session $(z=-0.44, p=.66)$.

\section{EEG results}

\section{ERP waveforms analysis}

The time-wise $2 \times 2$ waveform analyses on all electrodes with the factors Session and Language for the picture-naming task showed a Language $\times$ 

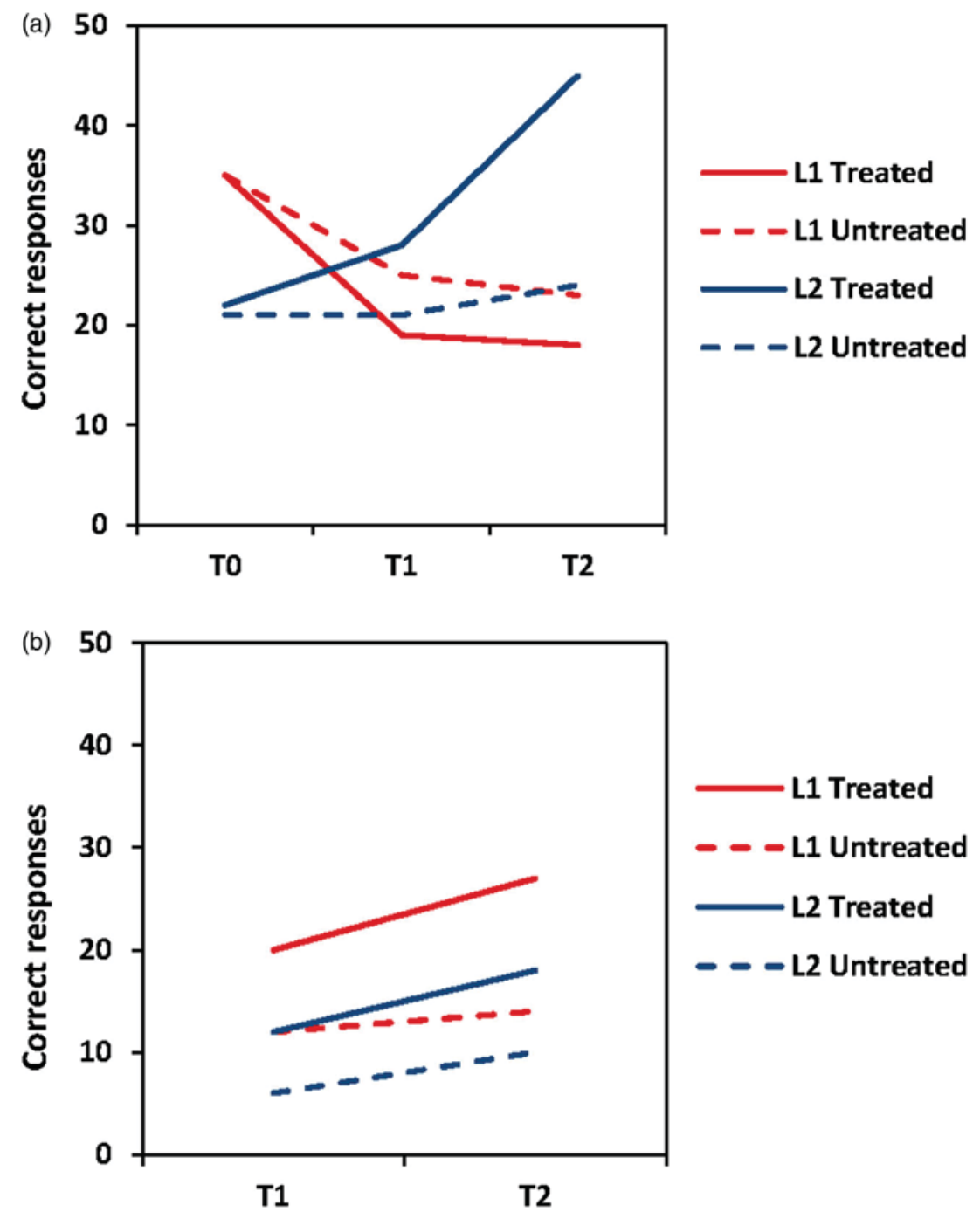

Figure 4. (a) Picture naming results at T0, T1 and T2: After the therapy (T2), the improvement in naming performances was found only for treated items in the treated language (L2). Detailed scores are as follow: $\mathrm{L} 1$ treated list: $\mathrm{T} 0=35, \mathrm{~T} 1=19, \mathrm{~T} 2=18 ; \mathrm{L} 1$ untreated list: $\mathrm{T} 0=35, \mathrm{~T} 1$ $=25, \mathrm{~T} 2=23 ; \mathrm{L} 2$ treated list: $\mathrm{T} 0=22, \mathrm{~T} 1=28, \mathrm{~T} 2=45 ; \mathrm{L} 2$ untreated list: $\mathrm{T} 0=21, \mathrm{~T} 1=21$, $\mathrm{T} 2=24$. (b) Word-picture verification results at $\mathrm{T} 1$ and $\mathrm{T} 2$ : In testing treated and untreated items separately, there is no main effect of Session and Language nor the interaction between Session and Language. Detailed scores are as follow: L1 treated list: T1 $=20, \mathrm{~T} 2=27$; L1 untreated list: $\mathrm{T} 1=12, \mathrm{~T} 2=14 ; \mathrm{L} 2$ treated list: $\mathrm{T} 1=12, \mathrm{~T} 2=18 ; \mathrm{L} 2$ untreated list: $\mathrm{T} 1=6, \mathrm{~T} 2=10$. 
Session interaction at left frontal electrodes from 200 to $260 \mathrm{~ms}$ and 550 to $600 \mathrm{~ms}$. See Figure 5(a) and (b).

The $2 \times 2$ waveform analyses of all electrodes for the word-picture verification task showed a Language $\times$ Session interaction at the frontal electrodes from -100 to $-80 \mathrm{~ms}$ (before the image presentation) and 310 to $360 \mathrm{~ms}$ after the image presentation.

The interpretation of the EEG data will however be based on the global dissimilarity analysis reported below.

\section{Global dissimilarity analysis}

- Picture naming: The $2 \times 2$ timeframe-wise analysis of global dissimilarity with factors Session (T1; T2) and Language (L1 and L2) showed a main effect of Session at 300-350 ms after the stimulus presentation. Critically, there was an interaction between the two factors at 540$600 \mathrm{~ms}$ after the stimulus presentation. See Figure 5(c).

Post-hoc analyses were conducted over the period showing the significant interaction 540 to $600 \mathrm{~ms}$ after the stimulus presentation to investigate the effect of session on each language separately. Over the period of interest, there was a significant effect of Session on the treated L2, $p<.001$, see Figure 5(c), and no significant effect of session for equivalent list in untreated $\mathrm{L} 1, p=.18$.

- Word-picture verification: The same design Session $(\mathrm{T} 1 ; \mathrm{T} 2) \times$ Language (L1; L2) as for the picture-naming task was used. There was a main effect of Session at $320-340 \mathrm{~ms}$ and $500-520 \mathrm{~ms}$ after the stimulus presentation. The main effect of Language was found from $20-120 \mathrm{~ms}, 300-420 \mathrm{~ms}$ and $460-600 \mathrm{~ms}$ after the stimulus presentation. No interaction was found between the factors Language and Session.

\section{DISCUSSION}

We examined the cross-language generalisation of intensive anomia lexicalphonological therapy on naming abilities in the second language (L2) of a bilingual Broca's aphasic patient presenting with severe word-finding difficulties and apraxia of speech.

Comparison between $\mathrm{T} 1$ and $\mathrm{T} 2$ shows an improvement in naming in the treated L2 but not in the untreated L1 language after intensive lexical-phonological therapy. Electrophysiological recording during naming corroborated these results by showing a topographic interaction between Session and Language at 540-600 ms, driven by an effect of session (before and after 
(a) Exemplar ERP waveforms

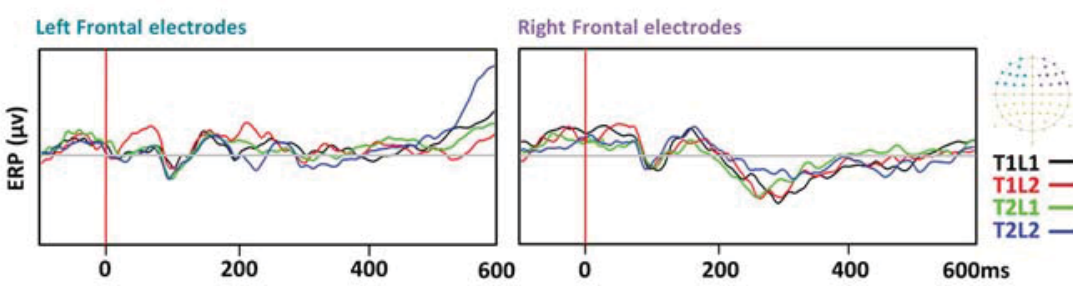

(b) Time-wise Language $\times$ Session interaction on the whole electrode montage

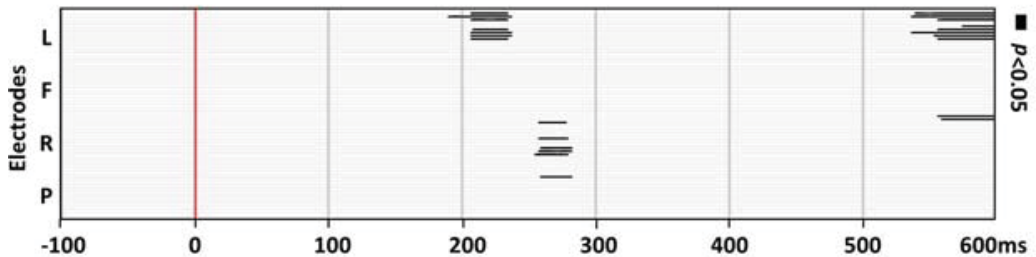

(c) Time-wise topographic Language $x$ Session interaction

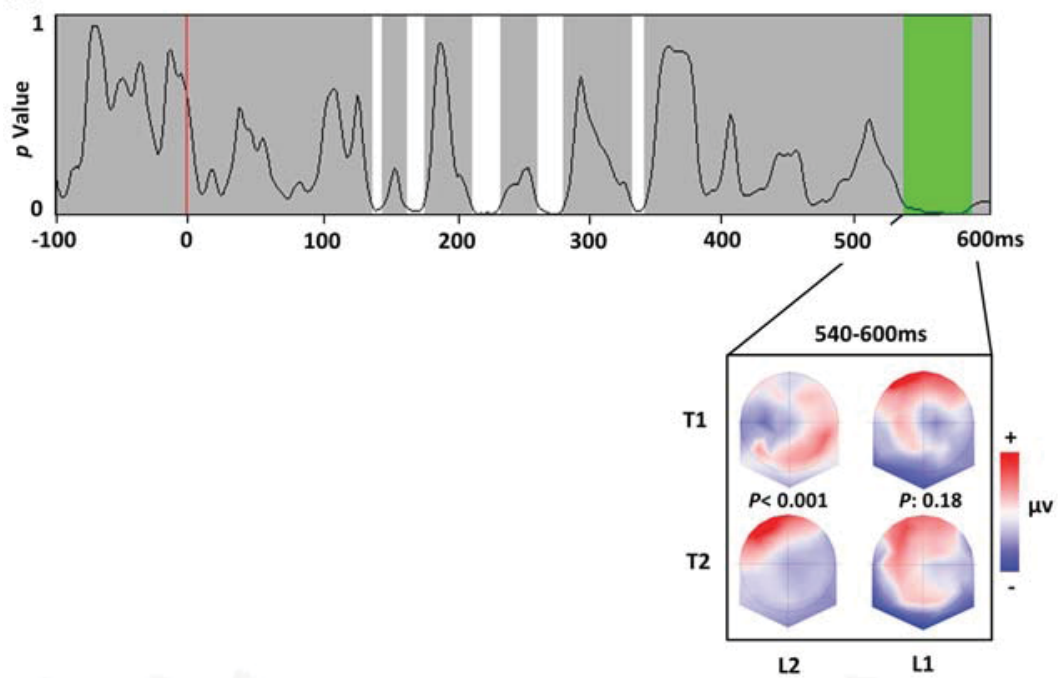

Figure 5. EEG analyses of picture-naming task: Waveforms (a) and (b) and topographic analyses (c): (a) Exemplar ERP waveforms of left and right frontal electrodes. (b) The time-wise $2 \times 2$ waveform analyses on all electrodes with the factors Session and Language for the picture naming task showing an interaction at left frontal electrodes from 200 to $260 \mathrm{~ms}$ and 550-600 ms. (c) The $2 \times 2$ time-frame wise analysis of global dissimilarity with factors Session and Language showing an interaction between the two factors at 540-600 ms after stimulus presentation. 
the therapy) on the ERP topography to the pictures in the treated L2 but not in the corresponding L1 stimuli. The absence of cross-language generalisation was confirmed by behavioural and EEG analyses. While a main effect of session in behavioural analysis could have been due to mere test-retest effects (habituation, modification in the attentional state between recordings, etc.) or caused by spontaneous recovery, the interpretation of the Language $x$ Session interaction term is unequivocal: the response to therapy in the treated L2 but not in the corresponding untreated L1 rules out that global, language unspecific effects accounted for our finding.

By contrast to the picture-naming task, the performance in the semantic word-picture verification task (a control semantic task) did not change at T2 either in the treated or untreated language. Consistently, electrophysiological topographic analyses of the word-picture verification task showed no interaction between the factors Session and Language. However, according to several models of lexical access (Costa et al., 2000) the semantic, but not the lexical system, is shared between both languages. Since therapy was focused at the lexical-phonological level, we did not expect that the effects of lexical-phonological therapy would transfer to the semantic system. This result should, however, be interpreted with caution since we cannot exclude type 2 error due to data insensitivity. Hence, a global ERP pattern modulation specific to treated items was observed only in the picture-naming task in the late 540-600 ms time-window.

According to previous estimates in healthy speakers (Indefrey, 2011; Indefrey \& Levelt, 2004) such a late time-window in the picture-naming task has been associated with phonetic encoding. Phonetic encoding is the stage of word production when articulatory gestures are generated from an abstract phonological code. In brain-damaged speakers, the phonological-phonetic encoding process, during which an abstract phonological code is transformed to a phonetic plan, has been suggested to be involved from around $400 \mathrm{~ms}$ after the picture presentation (Laganaro, Python, \& Toepel, 2013). Laganaro et al. (2013) found that in aphasic patients who produced specifically phonological and/or phonetic errors, ERPs to picture naming diverged from healthy control subjects after $400 \mathrm{~ms}$ post-picture onset. In another paper, by comparing the ERP to picture naming in two groups of anomic patients (with, respectively, predominantly semantic or phonological errors) with a healthy control group, Laganaro et al. (Laganaro, Morand, \& Schnider, 2009) showed different waveform amplitude and topographic maps at $\sim 100-250 \mathrm{~ms}$ only in the group with semantic errors, and different waveform amplitude and topographic maps at $\sim 300-450 \mathrm{~ms}$ only in the group with phonological errors; the waveforms and topographic maps were comparable to a healthy group out of these time windows. This finding accounts for the same languageprocessing pathway in aphasic patients with brain lesions and healthy subjects. However, in these previous studies, the time-course of divergent ERPs was 
compared to that of healthy speakers, therefore reflecting the dynamics of the controls, while aphasic patients had much longer production latencies. Accordingly, because of longer latencies in our patient's speaking onset, it is also probable that the effects that we found at the latency of $540-600 \mathrm{~ms}$, i.e., $100 \mathrm{~ms}$ later than in previous estimates, were also related to phonological-phonetic processes.

Because ERP topographic modulations necessarily follow from modifications in the configuration of neural generators, our results indicate that the lexical-phonological therapy modified qualitatively the brain networks engaged in phonetic encoding (and probably the phonological-phonetic process). More specifically, the therapy modified the brain networks engaged during naming in the treated but not in the untreated language. ERP change patterns after aphasia therapy have been rarely mentioned in the literature. We are aware of two studies: in four monolingual aphasic patients, Laganaro et al. (Laganaro, Morand, Schwitter, Zimmermann, \& Schnider, 2008) showed that post-treatment increased abnormal amplitude of ERP to picture naming, and different topographic map distribution (in comparison to the control group) occurs in the time windows corresponding to the impaired process, i.e., lexical-phonologic or lexical-semantic. The authors address these changes as "re-learning" of the process. However, after the treatment, in time windows corresponding to unimpaired processes, the ERP changed towards normalisation. In addition, Pulvermüller et al. (Pulvermüller, Hauk, Zohsel, Neininger, \& Mohr, 2005) suggested a post-treatment enhanced negativity in the ERP to words around $250-300 \mathrm{~ms}$ after word presentation in monolingual aphasic patients as an index of recovery from aphasia. They found that the ERP to pseudowords did not change after therapy, suggesting that change at this time window corresponds to lexico-semantic processes.

The absence of transfer of the effect of therapy to the untreated items (item-specificity of lexical-phonological therapy) is compatible with the discrete view which states that in the process of picture naming only the phonological segments of the selected word are activated and the activation of non-selected lexical representations does not spread to their phonological segments. In addition, the absence of CLG could be explained by the model of Costa et al. (2000) on language-specific lexical selection, which proposes that the activation of the shared semantic system spreads only to the lexical representations of the languages in use. Alternatively, the absence of CLG can also be accounted for by the fact that (1) the patient had a high proficiency in L2 and her main language exposure was in her L2 prior to the stroke, and (2) the etymological roots of the words in the two languages were mostly distinct.

Based on the Revised Hierarchical model, the link between lexicons is stronger from L2 to L1 than from L1 to L2 (Kroll \& Stewart, 1994); thus, 
in low proficient bilinguals, $\mathrm{L} 2$ is more dependent on borrowings from L1, while L2 in high proficient bilinguals is relatively independent of L1. In this regard, since our patient was highly proficient in L2, the therapy in L2 did not increase the access to L1 because the highly mastered L2 was quite independent from L1. Supporting this hypothesis, some authors have suggested that the patients with low L2 proficiency benefit from CLG after therapy in the less dominant L2 (Edmonds \& Kiran, 2006; Gil \& Goral, 2004). In contrast, another group of studies report no generalisation from the low proficient treated language to the non-treated high proficient L1: Miertsch et al. (2009) have found no generalisation from their patient's treated L3 to the stronger non-treated L1, while a CLG from the treated L3 to the non-treated L2 was found. The authors proposed that the absence of CLG to the untreated L1 (which was the main language of the environment) followed from the performance of the L1 being already high in the first assessment. Goral et al. (Goral, Rosas, Conner, Maul, \& Obler, 2012) examined a case of a multilingual aphasic patient (L1: Spanish, L2: German, L3: French and L4: English) and used a therapy in L1 and L4. They found no generalisation from L1 (again, the main language of the environment) to the other languages. In addition, after therapy in the less proficient language, L4, they observed no improvement in picture naming in untreated languages.

The absence of generalisation of the lexical-phonological therapy to the untreated language could also be explained by the fact that the patient's two languages (Persian and French) were different from each other at the level of the phonology, morphology, lexis and syntax. CLG has indeed been shown to be facilitated by a high degree of linguistic structure similarity between the treated and untreated languages. It has been suggested that transfer of the effect of therapy is expected at the level of shared linguistic structures in the two languages (Paradis, 1993) and thus CLG cannot take place between languages which do not share common structures (Kurland \& Falcon, 2011).

In contrast to our results for an absence of CLG to untreated L1, Marangolo et al. (2009) used a 6-month phonological therapy in L2 in a bilingual (Flemish/Italian) aphasic patient and found that CLG resulted in a parallel recovery of both languages after the therapy. This positive result possibly followed from the fact that the authors used a long duration therapy (6 months), whereas the intervention was much shorter in our study (4 weeks). Functional MRI recordings before and after two weeks of therapy in L2 confirmed their behavioural results by showing that the same brain regions were functioning for both languages before and after two weeks of the therapy.

Although we did not test directly for a difference between the effect of therapy on the treated vs. untreated items in L1 and L2, we did not find any main effect of Session nor Language $\mathrm{x}$ Session interaction for the 
untreated items. This result suggests that there were no cross-item generalisation of the therapy.

The lack of generalisation of the lexical-phonological therapy on the naming of untreated items in L2 corroborates current evidence that phonological treatment is item-specific. Hickin, Best, Herbert, Howard, and Osborne (2002) and Lorenz and Zieglerb (2009) found that phonological therapy for anomia in monolingual aphasic patients improved only the treated items and did not generalise to untreated items. Hickin et al. (2002) suggested that the effects of the therapy were item-specific because phonological therapy focuses on the "output form of the individual word".

In support of the fact that the phonological therapy improved only phonology and not semantic processing, there were neither main effects of Language and Session nor an interaction between Language and Session in the wordpicture verification task. The EEG result further supported this finding by showing no Language $\mathrm{x}$ Session topographic interaction. Electrophysiologically, we also found short-lived main effects of Session at 320-340 ms and $500-520 \mathrm{~ms}$ post-stimulus onset. As discussed above, main effects of session could be due to differences between the two recording sessions unrelated to the therapy and are thus difficult to interpret. Although we cannot exclude that the phonological therapy had a global effect on semantic processing in both L1 and L2 (more importantly on L1), the word-picture verification task relies less on phonological processing (Marshall, Pound, White-Thomson, \& Pring, 1990) and this hypothesis is thus unlikely.

Improvement of global score of language function only in the untreated language (L1) can be explained by different mechanisms: spontaneous recovery, generalisation of the effect of therapy, as well as the effect of language of environment. Spontaneous recovery consists of a series of physiological changes in the patient's brain taking place in the first weeks immediately after the onset of aphasia and generally occurring during the first three months after the stroke onset. Although the exact period which can be referred to as spontaneous recovery remains controversial (Gil \& Goral, 2004), in the beginning of the chronic phase, after three months post-lesion, spontaneous recovery generally slows down. In bilingual aphasic patients, spontaneous recovery can lead to both parallel and non-parallel recovery (Nilipour \& Ashayeri, 1989). Non-parallel spontaneous recovery of languages can be caused by differences in lexical systems of these languages in addition to complete or partial inhibition of one language during the activation of other languages (Green \& Price, 2001). Differentiation between spontaneous recovery and therapy-induced improvement is not well studied because eliminating therapy programmes after aphasia onset in order to study spontaneous recovery is not ethically acceptable (Basso et al., 2011). However, as stated before, spontaneous recovery decreases gradually around three months after the stroke. Although the treatment programme for this patient was performed 
after three months post-onset of aphasia, one cannot draw definite conclusions on the role of spontaneous recovery in our patient's improvement of global score of clinical aphasia assessment in L1. On the other hand, because there was no improvement in word-picture verification, the improvement of global score should be interpreted with caution. Therefore, in our patient it is difficult to determine to what extent the recovery of global score of the untreated language is related to phonological therapy in L2. In this regard, multiple evaluations after the therapy could have helped clarify the origin of this improvement. Unfortunately, the patient did not participate to further evaluations after the therapy.

We would further note that, before the therapy, two baseline tests of picture naming ( $\mathrm{T} 0$ and $\mathrm{T} 1$, one week apart) were conducted. These comparisons revealed that, at the beginning of the training, the naming performance was rather unstable; however, given that there was only one week between T0 and $\mathrm{T} 1$ and that the amplitude of the variations were small, we interpret these fluctuations as being primarily due to test-retest effects rather than as a consequence of spontaneous recovery. In addition, as detailed, the significant change in performance was specific to the treated L2, and accompanied by specific electrophysiological modulations only for treated items.

\section{CONCLUSION}

Our behavioural and EEG results suggest that intensive lexical-phonological therapy in L2 in a highly proficient bilingual aphasic patient with two etymologically different languages might be language-specific. The present results should however be considered as preliminary since the study focused on only a single case. A control condition in our patient (which would be to use the same therapy in L1) was not possible due to fatigability. Further studies are necessary to interpret patterns of generalisation in bilingual aphasic patients.

\section{REFERENCES}

Abutalebi, J., \& Green, D. W. (2007). Bilingual language production: The neurocognition of language representation and control. Journal of Neurolinguistics, 20, 242-275.

Aglioti, S., Beltramello, A., Girardi, F., \& Fabbro, F. (1996). Neurolinguistic and follow-up study of an unusual pattern of recovery from bilingual subcortical aphasia. Brain, 119(Pt 5), 1551-1564.

Ansaldo, A. I., Marcotte, K., Scherer, L., \& Raboyeau, G. (2008). Language therapy and bilingual aphasia: Clinical implications of psycholinguistic and neuroimaging research. Journal of Neurolinguistics, 21, 539-557.

Ansaldo, A. I., \& Saidi, L. G. (2014). Aphasia therapy in the age of globalization: Cross-linguistic therapy effects in bilingual aphasia. Behav Neurol, 2014, 603085. 
Basso, A., Cattaneo, S., Girelli, L., Luzzatti, C., Miozzo, A., Modena, L., \& Monti, A. (2011). Treatment efficacy of language and calculation disorders and speech apraxia: A review of the literature. Eur J Phys Rehabil Med, 47, 101-121.

Bates, D., Maechler, M., Bolker, B., \& Walker, S. (2014). lme4: Linear mixed-effects models using Eigen and S4. In (1.1-6 ed.).

Brunet, D., Murray, M. M., \& Michel, C. M. (2011). Spatiotemporal analysis of multichannel EEG: CARTOOL. Comput Intell Neurosci, 2011, 813870.

Costa, A., Colomé, A., \& Caramazza, A. (2000). Lexical access in speech production: The bilingual case. Psicológica, 21, 403-437.

Croft, S., Marshall, J., Pring, T., \& Hardwick, M. (2011). Therapy for naming difficulties in bilingual aphasia: Which language benefits?. Int J Lang Commun Disord, 46, 48-62.

De Bot, K. (1992). A bilingual production model: Levelt's speaking model adapted. Applied Linguistics, 13, 1-24.

Dijkstra, T., \& van Heuven, W. J. B. (2002). The architecture of the bilingual word recognition system: From identification to decision. Bilingualism-Language and Cognition, 5, 175-197.

Edmonds, L. A., \& Kiran, S. (2006). Effect of semantic naming treatment on crosslinguistic generalization in bilingual aphasia. Journal of Speech Language and Hearing Research, 49, 729-748.

Faroqi-Shah, Y., Frymark, T., Mullen, R., \& Wang, B. (2010). Effect of treatment for bilingual individuals with aphasia: A systematic review of the evidence. Journal of Neurolinguistics, 23, 319-341.

Gil, M., \& Goral, M. (2004). Nonparallel recovery in bilingual aphasia: Effects of language choice, language proficiency, and treatment. International journal of bilingualism, 8 , $191-219$.

Gollan, K. H., Montoya, R. I., Fennema-Notestine, C., \& Morris, S. K. (2005). Bilingualism affects picture naming but not picture classification. Memory \& Cognition, 33, 1220-1234.

Goral, M., Levy, E. S., \& Kastl, R. (2007). Cross-language treatment generalisation: A case of trilingual aphasia. Aphasiology, 103, 203-204.

Goral, M., Rosas, J., Conner, P. S., Maul, K. K., \& Obler, L. K. (2012). Effects of language proficiency and language of the environment on aphasia therapy in a multilingual. J Neurolinguistics, 25, 538-551.

Green, D., \& Price, C. (2001). Functional imaging in the study of recovery patterns in bilingual aphasia. Bilingualism: Language and Cognition, 4, 191-201.

Grosjean, F. (2004). Studying bilinguals: Methodological and conceptual issues. In The handbook of bilingualism (pp. 32-63). Oxford: Blackwell Publishing Ltd.

Guthrie, D., \& Buchwald, J. S. (1991). Significance testing of different potentials. Psychophysiology, 28, 240-244.

Hermans, D., Bongaerts, T., de Bot, K., \& Schreuder, R. (1998). Producing words in a foreign language: Can speakers prevent interference from their first language? Bilingualism: Language and Cognition, 1, 213-229.

Hickin, J., Best, W., Herbert, R., Howard, D., \& Osborne, F. (2002). Phonological therapy for word-finding difficulties: A re-evaluation. Aphasiology, 16, 981-999.

Hinckley, J. (2003). Picture naming treatment in aphasia yields greater improvement in L1. Brain and Language, 87, 171-172.

Howard, D., Patterson, K., Franklin, S., Orchard-Lisle, V., \& Morton, J. (1985). Treatment of word retrieval deficits in aphasia. A comparison of two therapy methods. Brain, 108(Pt 4), $817-829$.

Indefrey, P. (2011). The spatial and temporal signatures of word production components: A critical update. Frontiers in Psychology, 2, 255. doi:10.3389/fpsyg.2011.00255

Indefrey, P., \& Levelt, W. J. M. (2004). The spatial and temporal signatures of word production components. Cognition, 92, 101-144. 
Kiran, S., Sandberg, C., Gray, T., Ascenso, E., \& Kester, E. (2013). Rehabilitation in bilingual aphasia: Evidence for within and between-language generalization. Am J Speech Lang Pathol, 22, S298-S309.

Koenig, T., Kottlow, M., Stein, M., \& Melie-Garcia, L. (2011). Ragu: a free tool for the analysis of EEG and MEG event-related scalp field data using global randomization statistics. Comput Intell Neurosci, 2011, 938925.

Koenig, T., \& Melie-Garcia, L. (2010). A method to determine the presence of averaged eventrelated fields using randomization tests. Brain Topography, 23, 233-242.

Kohnert, K. (2004). Cognitive and cognate-based treatments for bilingual aphasia: A case study. Brain and Language, 91, 294-302.

Kohnert, K. (2009). Cross-language generalization following treatment in bilingual speakers with aphasia: A review. Seminars in Speech and Language, 30, 174-186.

Kroll, J., \& Stewart, E. (1994). Category interference in translation and picture naming: Evidence for asymmetric connections between bilingual memory representations. Journal of Memory and Language, 33, 149-174.

Kuhn, M., Weston, S., Wing, J., Forester, J., \& Thaler, T. (2013). Contrast: A collection of contrast methods. In (0.19 ed.).

Kurland, J., \& Falcon, M. (2011). Effects of cognate status and language of therapy during intensive semantic naming treatment in a case of severe nonfluent bilingual aphasia. Clinical Linguistics \& Phonetics, 25, 584-600.

Laganaro, M., Di Pietro, M., \& Schnider, A. (2003). Computerised treatment of anomia in chronic and acute aphasia: An exploratory study. Aphasiology, 17, 709-721.

Laganaro, M., Di Pietro, M., \& Schnider, A. (2006). Computerised treatment of anomia in acute aphasia: Treatment intensity and training size. Neuropsychological Rehabilitation, 16, $630-640$.

Laganaro, M., Morand, S., \& Schnider, A. (2009). Time course of evoked-potential changes in different forms of anomia in aphasia. Journal of Cognitive Neuroscience, 21, 1499-1510.

Laganaro, M., Morand, S., Schwitter, V., Zimmermann, C., \& Schnider, A. (2008). Normalisation and increase of abnormal ERP patterns accompany recovery from aphasia in the postacute stage. Neuropsychologia, 46, 2265-2273.

Laganaro, M., Python, G., \& Toepel, U. (2013). Dynamics of phonological-phonetic encoding in word production: Evidence from diverging ERPs between stroke patients and controls. Brain and Language, 126, 123-132.

Lehmann, D., Ozaki, H., \& Pal, I. (1987). EEG alpha map series: Brain micro-states by spaceoriented adaptive segmentation. Electroencephalography and Clinical Neurophysiology, 67, $271-288$.

Lehmann, D., \& Skrandies, W. (1980). Reference-free identification of components of checkerboard-evoked multichannel potential fields. Electroencephalography and Clinical Neurophysiology, 48, 609-621.

Levelt, W., Roelofs, A., \& Meyer, A. (1999). A theory of lexical access in speech production. Behavioral and Brain Sciences, 22.01, 1-38.

Lorenz, A., \& Zieglerb, W. (2009). Semantic vs. word-form specific techniques in anomia treatment: A multiple single-case study. Journal of Neurolinguistics, 22, 515-537.

Lucas, T. H., 2nd, McKhann, G. M., 2nd, \& Ojemann, G. A. (2004). Functional separation of languages in the bilingual brain: A comparison of electrical stimulation language mapping in 25 bilingual patients and 117 monolingual control patients. Journal of Neurosurgery, 101, 449-457.

Marangolo, P., Rizzi, C., Peran, P., Piras, F., \& Sabatini, U. (2009). Parallel recovery in a bilingual aphasic: A neurolinguistic and fMRI study. Neuropsychology, 23, 405-409.

Marshall, J., Pound, C., White-thomson, M., \& Pring, T. (1990). The use of picture/word matching tasks to assist word retrieval in aphasic patients. Aphasiology, 4, 167-184. 
Meinzer, M., Obleser, J., Flaisch, T., Eulitz, C., \& Rockstroh, B. (2007). Recovery from aphasia as a function of language therapy in an early bilingual patient demonstrated by fMRI. $\mathrm{Neu}$ ropsychologia, 45, 1247-1256.

Miertsch, B., Meisela, J. M., \& Isel, F. (2009). Non-treated languages in aphasia therapy of polyglots benefit from improvement in the treated language. Journal of Neurolinguistics, $22,135-150$.

Murray, M. M., Brunet, D., \& Michel, C. M. (2008). Topographic ERP analyses: A step-by-step tutorial review. Brain Topography, 20, 249-264.

Nilipour, R. (1988). Bilingual aphasia in Iran: A preliminary report. Journal of Neurolinguistics, $3,185-232$

Nilipour, R., \& Ashayeri, H. (1989). Alternating antagonism between two languages with successive recovery of a third in a trilingual aphasic patient. Bilingualism and Neurolinguistics, $36,23-48$.

Paradis, M. (1993). Bilingual aphasia rehabilitation. In Foundations of aphasia rehabilitation (pp. 413-419). Oxford: Pergamon Press.

Paradis, M. (1998). Language and communication in multilinguals. In Handbook of neurolinguistics (pp. 417-430). San Diego, CA: Academic Press.

Paradis, M., \& Libben, G. (1987). The assessment of bilingual aphasia. Hillsdale, N.J: Lawrence Erlbaum Associates.

Parker Jones, O., Green, D. W., Grogan, A., Pliatsikas, C., Filippopolitis, K., Ali, N., ... Price, C. J. (2012). Where, when and why brain activation differs for bilinguals and monolinguals during picture naming and reading aloud. Cerebral Cortex, 22, 892-902.

Peterson, R., \& Savoy, P. (1998). Lexical selection and phonological encoding during language production: Evidence for cascaded processing. Journal of Experimental Psychology: Learning, Memory, and Cognition, 24, 539-557.

Pillon, D., \& de Partz, M. P. (2005). A direct processing route to translate words from the first to the second language: Evidence from a case of a bilingual aphasic. Brain Lang, 95, 4041.

Pulvermüller, F., Hauk, O., Zohsel, K., Neininger, B., \& Mohr, B. (2005). Therapy-related reorganization of language in both hemispheres of patients with chronic aphasia. Neuroimage, $28,481-489$.

Roelofs, A. (1998). Lemma selection without inhibition of languages in bilingual speakers Bilingualism: Language and Cognition, 1, 94-95.

Team, R. D. C. (2008). R: A language and environment for statistical computing. Vienna, Austria: R Foundation for Statistical Computing. 